\title{
ELEMENTS DE PRECISION SUR LA SYSTEMATIQUE D'ESPECES ADVENTICES DUGENRE CORCHORUS L. (TILIACEAE) AU SENEGAL
}

\author{
Mbaye M.S. Noba K., Sarr R. S., Kane A., Sambou J.M. et BA Amadou Tidiane ${ }^{1}$. \\ 1 Département de Biologie Végétale, Faculté des Sciences et Techniques \\ Université Cheikh Anta DIOP, B.P. 5005 Dakar-Fann, SENEGAL. \\ Tel. (221) 824-80-01/824-03-02/824-21-03 Fax. (221) 824-21-03/824-37-14 E.mail ise@ @elecomplus-sn
}

\begin{abstract}
RESUME : Le genre Corchorus L. (Tiliaceae) comprend environ 40 espèces largement répandues sous les tropiques et est représenté au Sénégal par cinq espèces selon Berhaut: $C$. aestuans $L$., C. fascicularis Lam., C. olitorius $L$. avec deux variétés; C. olitorius var. olitorius $L$. et $C$. olitorius var. incisifolius Asch. \& Schw., C. tridens L. et C. trilocularis L.. Ces espèces présentent à la fois un intérêt économique et systématique. En effet outre leur statut d'adventices, elles ont des propriétés éthnobotaniques qui font que certaines espèces sont cultivées. Au plan systématique, elles appartiennent à un genre qui établit une transition entre deux tribus; Tiliae et Grewiae. Bien qu'ayant fait l'objet de plusieurs travaux, la détermination de ces espèces reste toujours difficile. En effet, les caractères reconnus comme les plus discriminants ne concernent que le fruit qui apparaît tard dans le développement de la plante L'étude des organes végétatifs et reproducteurs de la plante adulte a montré que la présence ou non des filaments capillaires à la base du limbe, la marge dentée ou lacérée des feuilles, l'ovaire triloculaire ou pentaloculaire, la section circulaire, hexagonale, pentagonale ou trigone du fruit, le sommet entier ou divisé du fruit, les graines contiguës ou séparées dans le fruit constituent autant de caractères qui permettent d'améliorer l'identification de ces espèces.
\end{abstract}

MOTS CLES : Corchorus L., Tiliaceae, Systématique.

\begin{abstract}
Corchorus $L$ is a genus includes nearly 40 species widelydistributed throughout the tropics and is represented in Sénégal by 5 species according to Berhaut : C. aestuans L.; C. fascicularis Lam., C. olitorius L. with 2 varieties C. olitorius var olitorius $L$. and C. olitorius var incisifolius Asch. \& Schw; C. tridens $L$. and C. trilocularis L.. Interest for these species is both economic and systematic. Indeed, beyond their weed statute and because of their ethnobotanical properties some species are cultivated. Besides, in the botanical point of view they belong to a genus which is a transition group between two tribes; Tiliae and Grewiae. Even works done on the genus, identification of these species is still difficult. In fact, the more discriminant characters put ahead concern those of the fruit, an organ which appears late in the plant life cycle. Investigations on vegetative and reproductive organs on adult plant showed that, the presence or absence of basal hair on the limb, the dentate or lacerate leaves, the ovary 3 or 5-celled, the circular or hexagonal or pentagonal or trigonal secttion of the fruit, the seeds contiguous or separate inside the fruit are additional characters which could better help to identify these species.
\end{abstract}

KEY WORDS : Corchorus L., Tiliaceae, Systematic.

\section{INTRODUCTION}

Le genre Corchorus L. appartient à la famille des Tiliaceae et renferme une quarantaine d'espèces. Il est représenté au Sénégal par cinq espèces (Berhaut, 1967) : C. aestuans L., C. fascicularis Lam., C. olitorius L. avec deux variétés C. olitorius var olitorius L. et C. olitorius var incisifolius Asch. \& Schw., $C$. tridens L. et $C$. trilocularis L..
Ces espèces présentent une grande importance économique et systématique.

Du point de vue économique, en plus de leur statut d'adventices des cultures tropicales (Merlier et Montégut, 1982 ; Le Bourgeois et Merlier, 1995 ;Johnson, 1997 ; Noba et Bâ, 1998 ; Mbaye. 1999) ces espèces présentent d'autres intérêts. En effet elles ont de nombreuses utilisations. Les 
feuilles de Corchorus L. sont riches en acides aminés essentiels, seule la méthionine y est présente en faible quantité (Fafunso \& Bassir, 1975). Les feuilles de $C$. olitorius L., C. aestuans L., C. tridens L., C. capsularis, $C$. depressus etc sont utilisées dans l'alimentation humaine sous le nom de Corètes, comme les Laitues et les Epinards. Les espèces du genre présentent également des propriétés émollientes et mucilagineuses (Baillon, 1886 ; Pursglove, 1968). Les tiges contiennent des fibres qui sont utilisées dans la fabrication de fils et cordes, de toiles d'emballage et dans la confection de sacs (Anonyme, 1991). C. olitorius L. est considéré comme un taxon médicinal du fait de ses graines qui sont utilisées en Inde comme purgatif et de ses feuilles qui sont toniques et diurétiques (Olivier-Bever, 1986). C. aestuans L. est également considéré comme un taxon médicinal puisque ses feuilles et ses racines sont utilisées en Afrique occidentale contre la blennorrhagie, tandis que ses fruits et ses graines soignent la colique et la pneumonie (Ayensu, 1978).

Toutes ces propriétés font que certaines espèces de Corchorus L. sont cultivées. C'est le cas de C. olitorius L. (Akoroda, 1988 ; Akoroda \& Akintobi, 1983 ;Akoroda \& Olufajo, 1981 ;Timpo \& Boateng, 1982)

En outre l'organisation et la forme du réceptacle floral, tantôt court, tantôt en colonne rencontrée chez diverses espèces font de ce genre un groupe de transition entre les Tiliae et les Grewiae, deux tribus de la famille des Tiliaceae (Baillon, 1886).

Pour leurs intérêts divers, ces espèces ont fait l'objet de plusieurs études parmi lesquelles celles de Roberty (1954), Hutchinson \& al (1958), Berhaut (1967) ; Epenhuijsen (1974), Merlier et Montégut (1982), Norman (1992), Le Bourgeois \& Merlier (1995), Mbaye (1999).

Il ressort de ces études que les caractères du fruit sont les plus discriminants. En effet les caractères morphologiques et anatomiques de l'appareil végétatif ainsi que ceux de la fleur présentent de très grandes ressemblances entre les espèces rendant ainsi difficile leur identification.

Le but de ce travail est de rechercher des caractères discriminants nouveaux afin d'améliorer la clé de détermination de ces espèces.

\section{MATERIELSETMETHODES}

Les observations de la plante adulte ont été réalisées sur des individus vivant dans des milieux variés et des échantillons conservés dans les herbiers de l'IFAN et du Département de Biologie Végétale par Berhaut, Madsen,
Seck, Kane Schneider, Bodian, Adam, Monod, Roberty, Naegele, De Waily, Tisserant, Garnier, Duong, Sarr, Thiam, Madsen, Raynal, Cornick, Giovannetti, Prost, Pitot, Nongonierma et Ronette entre autres.

Sur ces individus, des observations à l'œil nu ou à la loupe binoculaire ont été effectuées et ont porté sur :

-les caractères morphologiques qualitatifs de certains organes comme la tige (couleur, pilosité, port), la racine, la feuille (type, forme, couleur, pilosité, nervation), les stipules, les filaments capillaires basaux, la fleur (symétrie, préfloraison, diagramme), fruit (forme, aspects), la graine (forme, aspects, couleurs) ;

-les caractères morphologiques quantitatifs (dimensions) de certains organes feuilles, fleurs, fruit, graines.

Sur les feuilles sont entreprises des observations micromorphologiques portant sur le type et l'indice stomatique. Les stomates et les cellules épidermiques banales des épidermes supérieur et inférieur d'une dizaine de feuilles ont été dénombrés à raison de cinq comptages par face ce qui donne une cinquantaine de comptages par face.

Après avoir évalué le nombre de stomates et des cellules épidermiques banales par face et par espèce, nous avons calculé l'indice stomatique (IS) de chaque espèce qui représente la fréquence relative des stomates c'est-à-dire le nombre de stomate $\left(a_{1}\right)$ pour l'espèce 1 par le nombre total de cellules épidermiques de cette espèce $n_{1}\left(n_{1=} a_{1}+\right.$ $\left.\mathrm{b}_{1}\right), \mathrm{b}_{1}$ représentant le nombre de cellules épidermiques banales.

$$
I S=\frac{a_{1}}{a_{1}+b_{1}}
$$

\section{RESULTATS ET DISCUSSIONS}

\section{La feuille ( $\mathrm{Pl} . \mathrm{I})$}

L'étude macromorphologique de la feuille a porté sur les caractères du limbe et du pétiole. Au niveau du limbe, les observations ont porté sur la forme générale, le sommet, la base, la marge, la nervation, les stipules ainsi que la présence ou l'absence de filaments capillaires.

Le résultat de l'observation de ces différents caractères est résumé dans le tableau I. 
Elements de Precision sur la Systematique D'especes Adventices du Genre Corchorus L. (Tiliaceae) au Senegal.

Tableau I : Caractères de la feuille chez les espèces de Corchorus L. du Sénégal

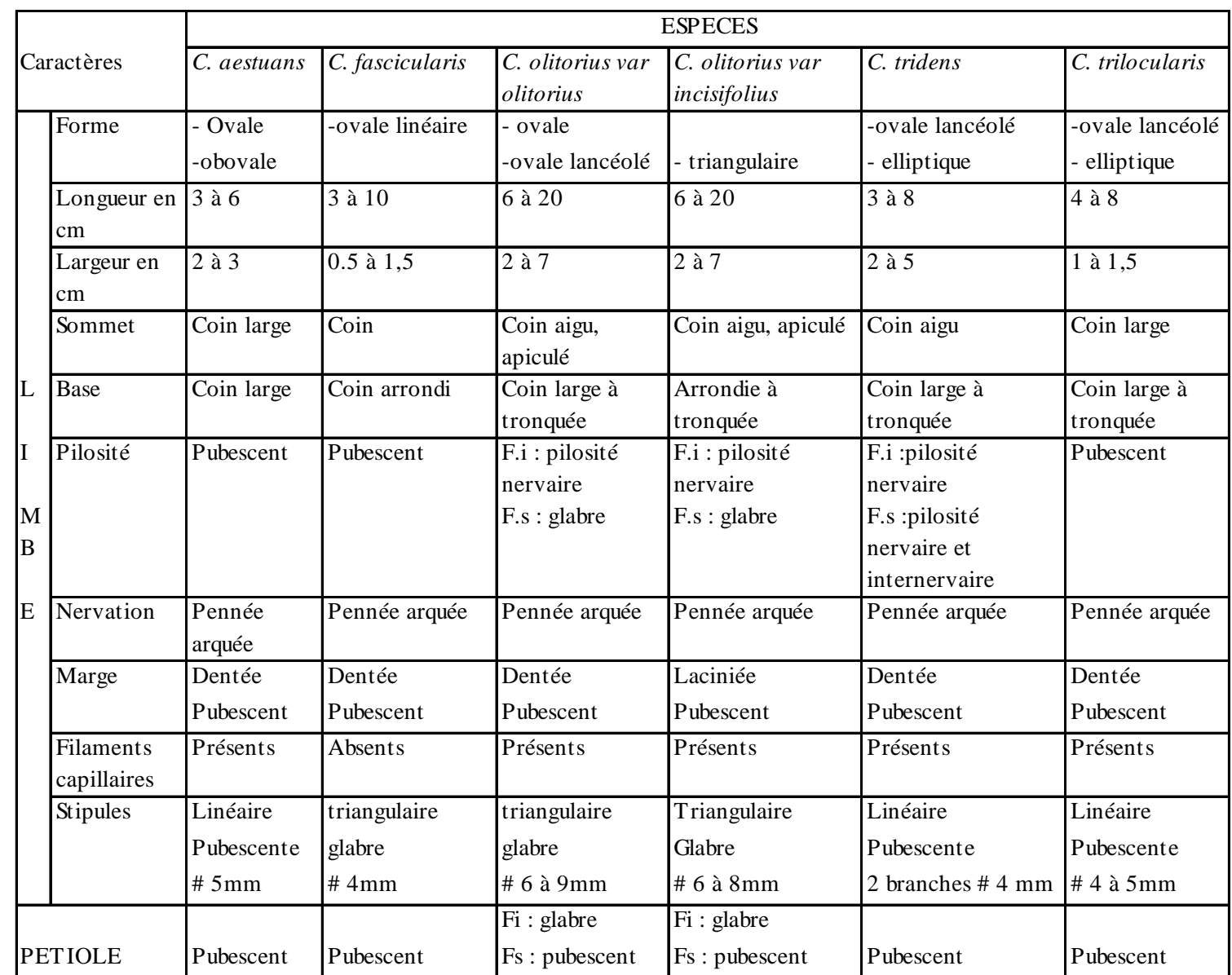

$\mathrm{Fi}=$ Face inférieure

Fs = Face supérieure

L'analyse de ce tableau montre que les feuilles de Corchorus L. présentent beaucoup de caractères communs. Cependant une observation plus attentive fait ressortir des caractères différentiels qui permettent une meilleure identification de ces espèces.

En effet, les filaments capillaires basaux (PL.I ; 3b) se rencontrent chez toutes les espèces sauf chez $C$. fascicularis Lam. (PL.I ; 2). Les feuilles de ces espèces sont généralement ovales à l'exception de celles de $C$. olitorius var olitorius L.. Le limbe est pubescent même si la localisation des poils est différente selon les espèces. Ainsi chez $C$. olitorius L. les poils ne sont présents que sur les nervures à la face inférieure contrairement aux autres espèces. La laciniation du limbe de C. olitorius var incisifolius Asch. \& Schw. est caractéristique de ce taxon (PL.I ; 3c) ; les autres ayant la marge du limbe régulièrement dentée. Le pétiole des deux variétés de $C$. olitorius L. n'est pubescent que sur le sillon situé à la face supérieure alors que chez les autres espèces le pétiole est pubescent sur
\# = environ

toute sa surface avec une densité légèrement différente en fonction des espèces.

Les stipules sont linéaires et pubescentes chez $C$. aestuans L., $C$. tridens L. et $C$. trilocularis L. alors qu'elles sont triangulaires et glabres chez les autres espèces.

L'étude micromorphologique de la feuille a porté sur les stomates (type et indice stomatiques) et sur le trichome

Il est reconnu que les types stomatiques et les caractéristiques des cellules épidermiques banales (PL. II, III et IV) ont une grande valeur taxonomique (Metcalfe et Chalk, 1950 ; Guyot ; 1966 ; Dupont, 1968 ; Gorenflot et Moreau, 1970 ; Gorenflot, 1971 ;Noba, 1990).

Pour le type stomatique, nous avons utilisé la classification de Metcalfe et Chalk (1950) qui distingue 4 types de stomates. 


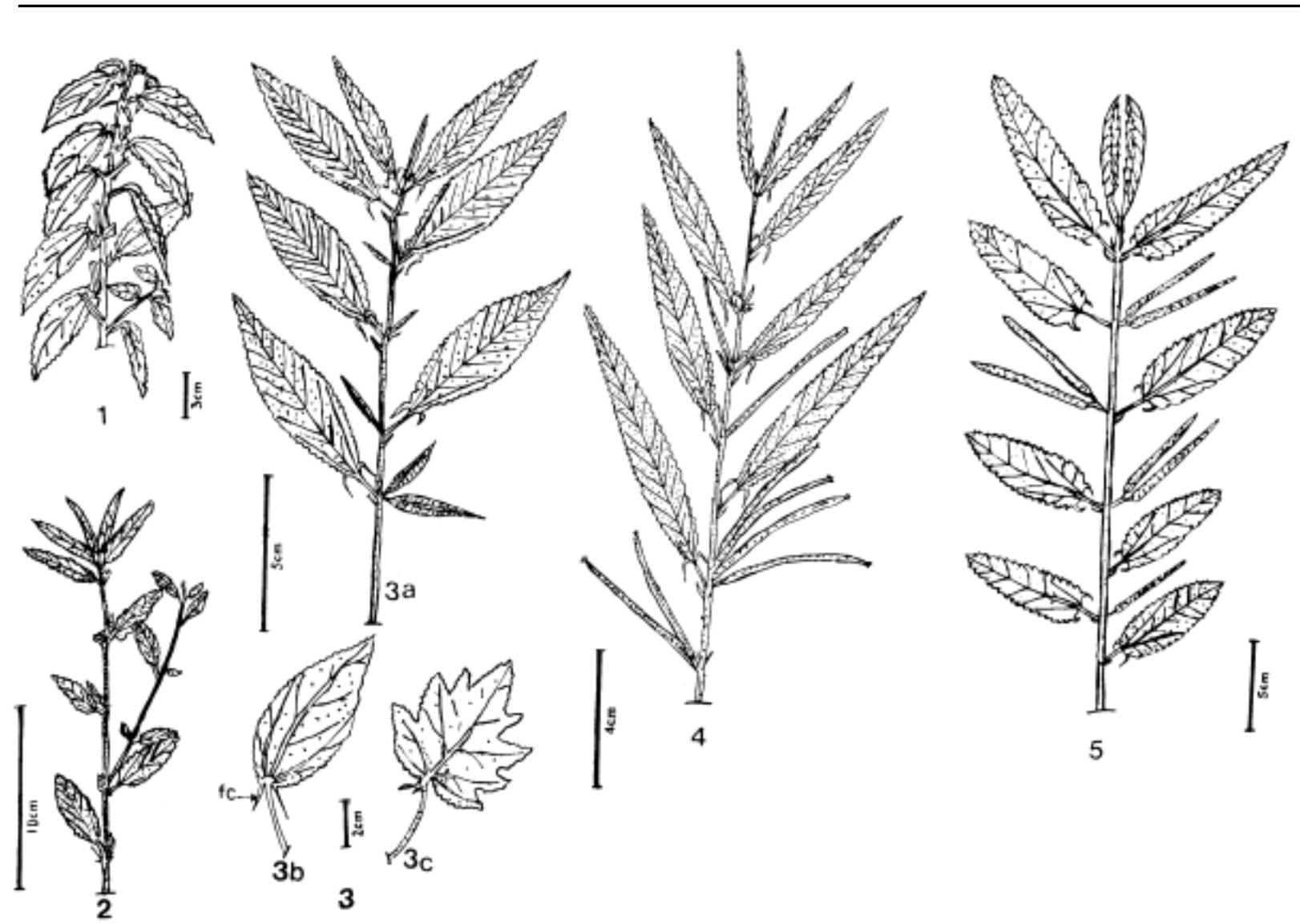

Planche I : Morphologie au stade adulte des espèces de Corchorus

Fig.1 - C. Aestuans; Fig.2 - C. Fascicularis; Fig.3 - C. olitorius; 3a - rameau 3b - Feuille de C. olitorius var. Olitorius; 3c - Feuille de C. olitorius var. incisifolius $\mathrm{fc}=$ filament capillaire basal Fig. $4-$ C. Tridens Fig. $5-$ C. trilocularis

Il ressort de notre étude que les espèces de Corchorus L. étudiées présentent le même type stomatique en l'occurrence le type anisocytique ou crucifère (PL.II ; 2). De même l'épiderme supérieur possède des cellules banales à parois latérales non sinueuses ni ondulées.

L'indice stomatique a une importance taxonomique moindre que le précédent. En effet si le premier (type stomatique) est plus ou moins constant pour chaque espèce, le second (indice stomatique) en revanche est variable en fonction des conditions du milieu (Timmerman, 1927 ; Salisbury, 1927 ; Metcalfe et Chalk, 1950 ; Noba, 1990 ; Noba \& al 1994).

L'étude quantitative de l'épiderme foliaire a donné les résultats consignés dans le tableau II:

Les indices stomatiques sont dans l'ensemble faibles (moins de $27 \%$ ) pour les espèces étudiées. Néanmoins, $C$. tridens L. a l'indice le plus faible $(14,03 \%)$ alors que $C$ trilocularis L. présente l'indice le plus élevé (26,95\%).
L'observation du trichome foliaire complète utilement celle de l'appareil stomatique (Moreau, 1979).

Merlier et Montégut (1982) ont proposé une classification des poils selon quatre critères : l'intensité, l'orientation des poils et leur diversité, la nature des poils et la localisation suivant les organes.

Chez les espèces Corchorus L. étudiées, il existe une homogénéité du type de poils. Le type unique de poils rencontré sur tous les organes pubescents est le type tuberculé simple à soubassement vésiculeux. Ces poils sont unicellulaires, unisériés et stellaires (PL.II ; 1).

En fonction de la disposition des poils épidermiques foliaires nous pouvons distinguer deux types:

-les poils situés sur les nervures ou poils nervaires rencontrés sur les faces supérieure et inférieure des feuilles de $C$. aestuans L., C. fascicularis Lam., C. tridens L. et $C$. trilocularis L. et sur la face inférieure des feuilles des deux variétés de $C$. olitorius $\mathrm{L}$. 

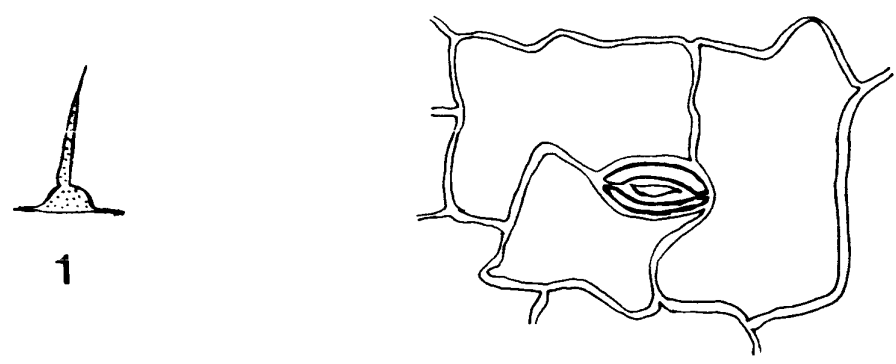

2

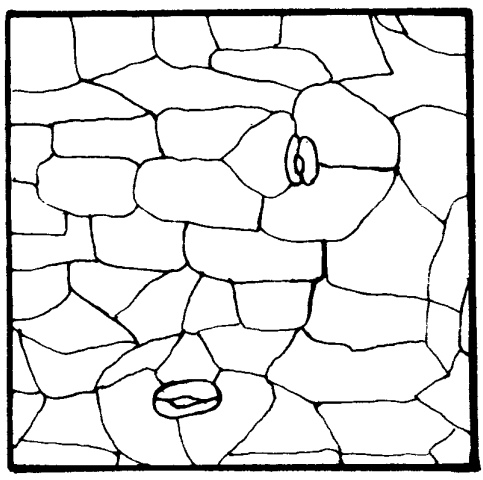

$3 a$

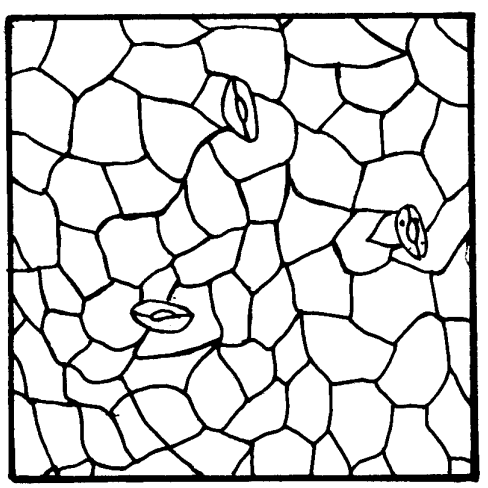

$4 a$

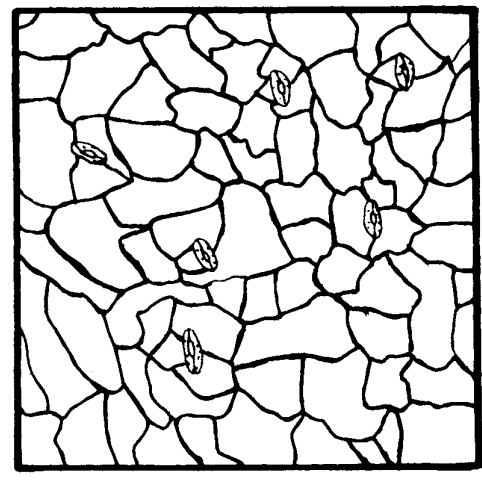

$3 b$

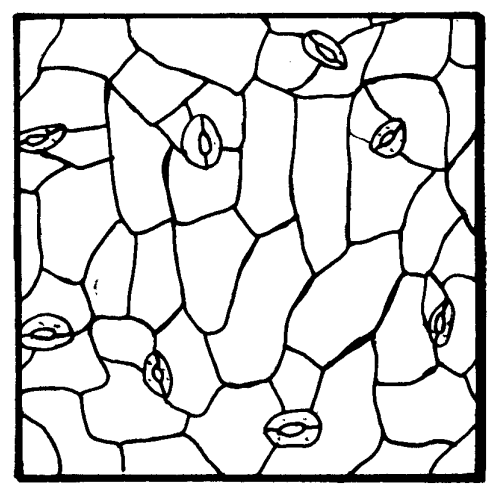

$4 b$

Planches II, III et IV : Structure de l'épiderme foliaire des six taxons

Fig.1 - Poil tuberculé simple à soubassement vésiculeux ; Fig.2 - Type anisocytique ; Fig.3 - Epiderme de $C$. aestuans ; Fig.4 - Epiderme de C. fascicularis 
M. S. MBAYE

Tableau II : Résultats de l'étude quantitative de l'épiderme des feuilles de Corchorus

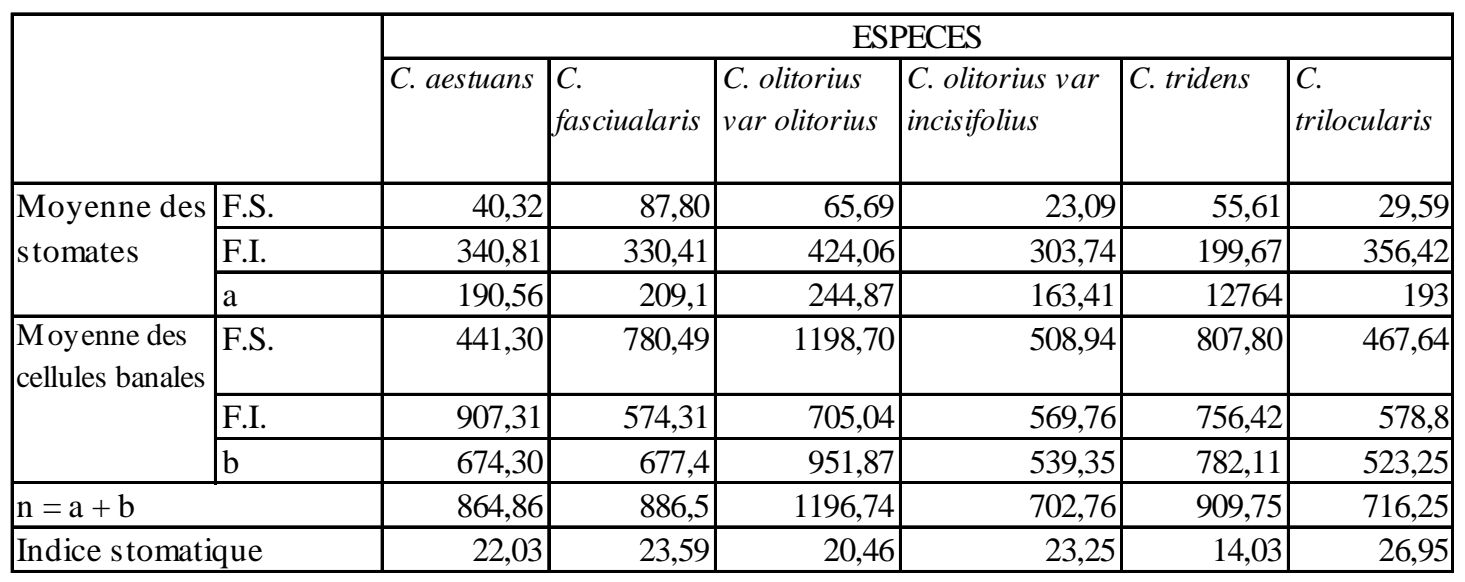

FS $=$ Face supérieure

FI = Face inférieure

$\mathbf{a}=$ moyenne des stomates sur les deux faces

$\mathbf{b}=$ moyenne des cellules banales sur les deux faces

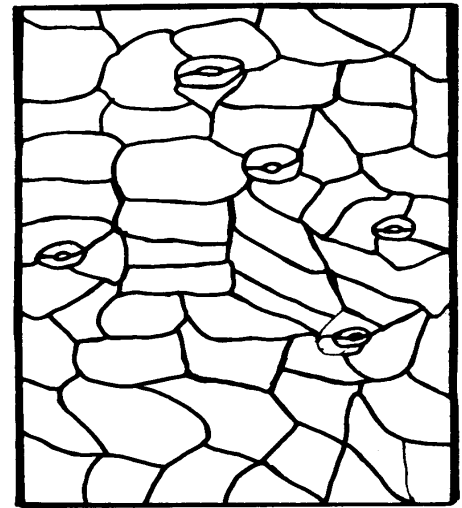

5 a

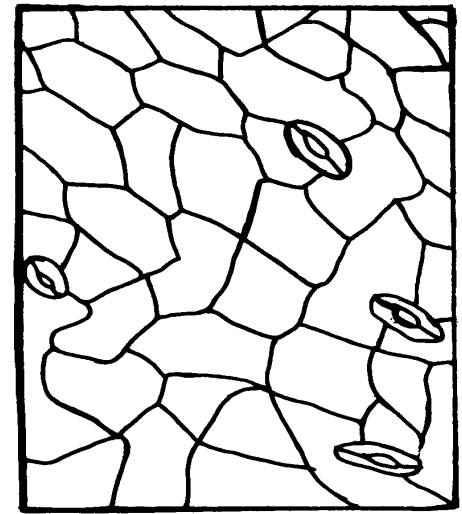

6 a

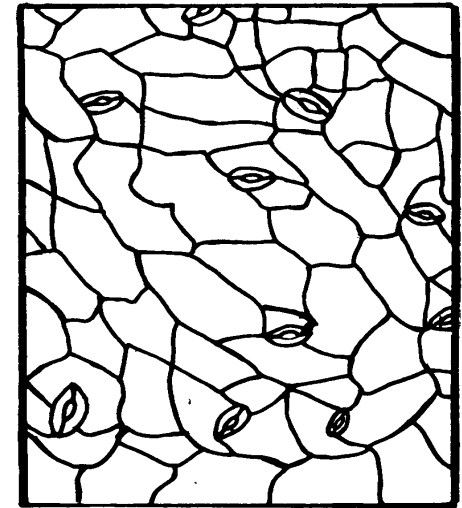

$5 b$

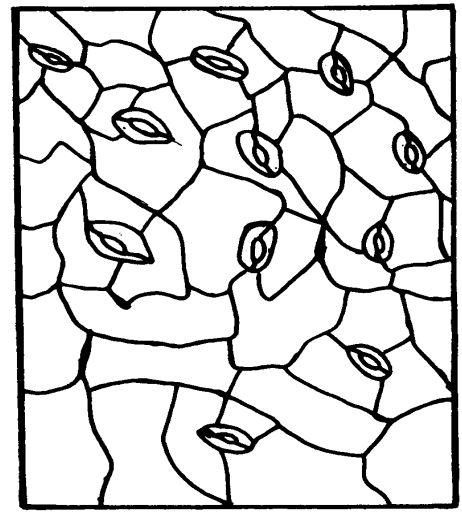

$6 b$

Fig.5 - Epiderme de C. olitorius var. olitorius; Fig.6 - Epiderme de C. olitorius var. incisifolius 


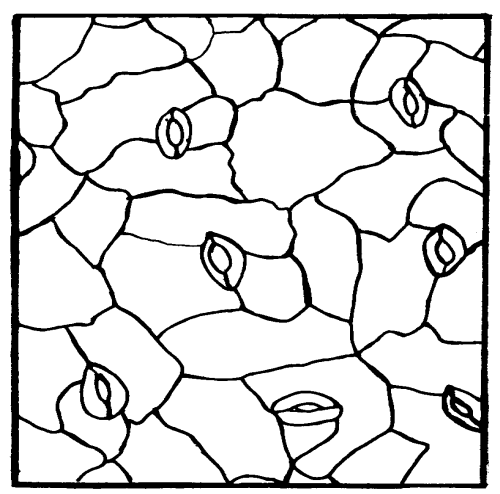

$7 \mathbf{a}$

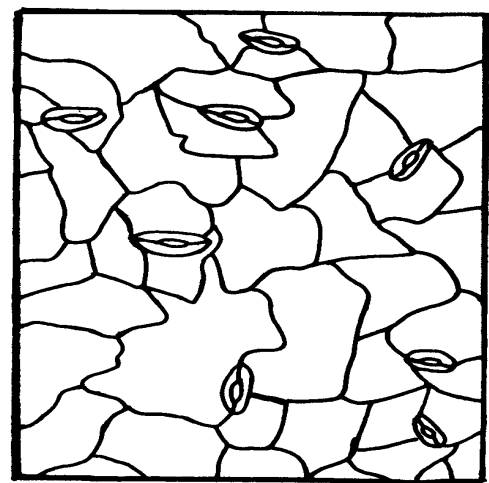

8 a

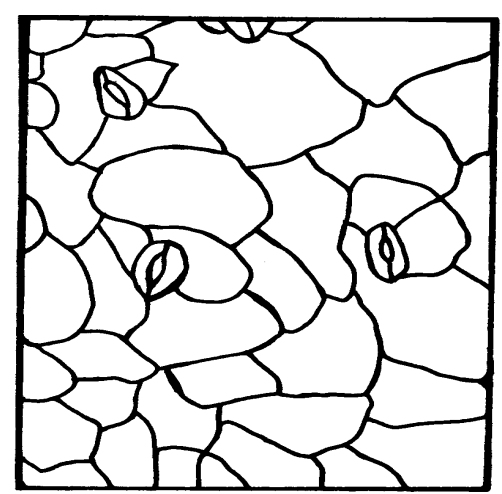

$7 b$

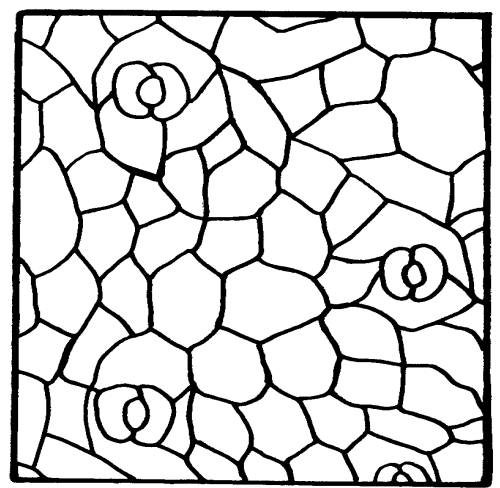

8b

Fig. 7 - Epiderme de C. tridens; Fig. 8 - Epiderme de C. trilocularis a - Epiderme inférieur b - Epiderme supérieur

-les poils internervaires observés sur les faces supérieure et inférieure des feuilles de C. aestuans L., C. fascicularis Lam. et $C$. trilocularis $\mathrm{L}$. et sur la face inférieure des feuilles de $C$. tridens L..

\section{-La fleur (PL. V)}

La fleur des espèces étudiées du genre Corchorus L. présente des pétales fendus au moins sur la moitié de leur longueur, des carpelles directement posés sur le réceptacle floral proéminent, fortement convexe ou plan, un ovaire pluriloculaire à placentation axile, des carpelles soudés et un calice à préfloraison valvaire. Elle est dépourvue de calicule, de staminodes, et possède des anthères biloculaires. L'observation de la fleur de ces espèces a donné les résultats suivants (Tableau III) :

La fleur de ces espèces présente de très grandes ressemblances. En effet, à l'exception de celle $C$. aestuans qui peut être tétra ou pentamère, la fleur des autres espèces est pentamère. La fleur hermaphrodite, le calice valvaire, l'ovaire supère sont autant de caractères constants chez ces espèces. Cependant en plus les différences de la mérie, certains caractères sont variables selon les espèces comme la préfloraison de la corolle qui est tordue chez C. aestuans (PL. V ; 5b) et imbriquée chez les autres espèces (PL. V ; 5a), l'ovaire triloculaire chez $C$ aestuans, $C$. tridens et $C$. trilocularis et pentaloculaire chez les autres.

\section{-Le fruit et la graine (PL. VI)}

Le fruit des Corchorus est une capsule, fusiforme à linéaire. Il est allongé, lisse ou strié. La capsule a deux à cinq valves selon les espèces et est à déhiscence loculicide ; elle est intérieurement et quelquefois transversalement séptée.

Les graines sont nombreuses, pendantes ou horizontales et albuminées

L'embryon est orthotrope ou courbé.

Les caractères du fruit et de la graine sont répertoriés dans le tableau IV. 
M. S. MBAYE

Tableau III : Caractères morphologiques de la fleur des différentes espèces de Corchorus L. étudiées.

\begin{tabular}{|c|c|c|c|c|c|c|}
\hline \multirow{2}{*}{$\begin{array}{l}\text { Caractères de la } \\
\text { fleur }\end{array}$} & \multicolumn{6}{|c|}{ ESPECES } \\
\hline & C. aestuans & $\begin{array}{l}\text { C. } \\
\text { fascicularis }\end{array}$ & $\begin{array}{l}\text { C. olitorius var } \\
\text { olitorius }\end{array}$ & $\begin{array}{l}\text { C. olitorius var } \\
\text { incisifolius }\end{array}$ & C. tridens & C. trilocularis \\
\hline \begin{tabular}{|l} 
Nombre et \\
disposition des fleurs
\end{tabular} & $\begin{array}{l}1 \text { à } 2 \text { opposées } \\
\text { au pétiole }\end{array}$ & $\begin{array}{l}3 \text { à } 8 \text { à la base } \\
\text { des feuilles }\end{array}$ & $\begin{array}{l}1 \text { à } 2 \text { opposées } \\
\text { au pétiole }\end{array}$ & $\begin{array}{l}1 \text { à } 2 \text { opposées } \\
\text { au pétiole }\end{array}$ & $\begin{array}{l}2 \text { à } 6 \text { opposées } \\
\text { au pétiole }\end{array}$ & $\begin{array}{l}1 \text { à } 3 \text { opposées } \\
\text { au pétiole }\end{array}$ \\
\hline Calice & $\begin{array}{l}4 \text { à } 5 \text { sépales } \\
\text { - Vert } \\
\text { - Valvaire }\end{array}$ & $\begin{array}{l}\text { - } 5 \text { sépales } \\
\text { - Vert } \\
\text { - Valvaire }\end{array}$ & $\begin{array}{l}\text { - } 5 \text { sépales } \\
\text { - Vert } \\
\text { - Valvaire }\end{array}$ & $\begin{array}{l}\text { - } 5 \text { sépales } \\
\text { - Vert } \\
\text { - Valvaire }\end{array}$ & $\begin{array}{l}\text { - } 5 \text { sépales } \\
\text { - Vert } \\
\text { - Valvaire }\end{array}$ & $\begin{array}{l}\text { - } 5 \text { sépales } \\
\text { - Vert } \\
\text { - Valvaire }\end{array}$ \\
\hline Corolle & $\begin{array}{l}4 \text { à } 5 \text { pétales } \\
\text { jaune orangé } \\
\text { Tordue }\end{array}$ & $\begin{array}{l}\text { - } 5 \text { pétales } \\
\text { jaunes } \\
\text { - Imbriquée }\end{array}$ & $\begin{array}{l}\text { - } 5 \text { pétales } \\
\text { jaunes } \\
\text { - Imbriquée }\end{array}$ & $\begin{array}{l}\text { - } 5 \text { pétales } \\
\text { jaunes } \\
\text { - Imbriquée }\end{array}$ & $\begin{array}{l}\text { - } 5 \text { pétales } \\
\text { jaunes } \\
\text { - Imbriquée }\end{array}$ & $\begin{array}{l}\text { - } 5 \text { pétales } \\
\text { jaunes } \\
\text { - Imbriquée }\end{array}$ \\
\hline Androcée & n étamines & n étamines & n étamines & n étamines & n étamines & n étamines \\
\hline Ovaire & $\begin{array}{l}\text { - Supère } \\
\text { - triloculaire } \\
\text { - n ovules }\end{array}$ & $\begin{array}{l}\text { - Supère } \\
\text { - 5-loculaire } \\
\text { - n ovules }\end{array}$ & $\begin{array}{l}\text { - Supère } \\
\text { - 5-loculaire } \\
\text { - n ovules }\end{array}$ & $\begin{array}{l}\text { - Supère } \\
\text { - 5-loculaire } \\
\text { - n ovules }\end{array}$ & $\begin{array}{l}\text { - Supère } \\
\text { - triloculaire } \\
\text { - n ovules }\end{array}$ & $\begin{array}{l}\text { - Supère } \\
\text { - triloculaire } \\
\text { - n ovules }\end{array}$ \\
\hline
\end{tabular}

Le fruit en capsule cylindrique, la déhiscence loculicide, la grande quantité de graines sont des caractères communs entre ces espèces. A coté, il existe des caractères distinctifs au niveau des fruits et des graines.

Le fruit n'est pubescent que chez C. trilocularis L. (PL.VI ;7) et dans une moindre mesure chez $C$. fascicularis Lam. (PL. VI ; 5). Le sommet du fruit est droit et entier chez toutes les espèces sauf chez $C$. aestuans L. (PL.VI; 9) et $C$. tridens L. (PL.VI ; 5) où il est terminé par trois dents divergentes.

Les graines sont polyédriques chez $C$ aestuans L., $C$. fascicularis Lam. et $C$. trilocularis L. alors qu'elles sont pyramidales chez $C$. olitorius L. et trapézoïdales chez $C$. tridens. L.. Ces graines sont contiguës dans le fruit chez $C$ aestuans L. et C. fascicularis Lam. alors qu'elles sont séparées chez les autres.

C. aestuans L., C. fascicularis Lam., C. olitorius var olitorius L., C. olitorius var incisifolius Asch. \& Schw., $C$. tridens L. et $C$. trilocularis L. présentent des ressemblances qui font qu' elles forment un complexe dans lequel les limites entre taxons ne sont pas toujours nettement définies. L'étude morphologique et anatomique des appareils végétatif et reproducteur de ces espèces a permis d'apporter des éléments nouveaux qui précisent la clé de détermination de ces espèces.

Les caractères discriminants nouveaux identifiés au cours de cette étude sont réunis dans le tableau synoptique (Tableau V) :

L'analyse de ce tableau montre que malgré une très grande ressemblance, ces espèces présentent des différences macroscopiques pouvant améliorer leur identification.

Les principales différences macromorphologiques au stade adulte sont les suivantes :

- toutes les feuilles adultes possèdent des filaments capillaires basaux exceptées celles de $C$. fascicularis Lam. (PL.I ; 2) ;

- le limbe est régulièrement denté à part celui de $C$. olitorius var incisifolius Asch. \& Schw. qui est lacéré (PL.I ; 3c) ;

- $\quad$ l'ovaire est triloculaire chez C. aestuans L., C. tridens L. et $C$. trilocularis L. et pentaloculaire chez les autres espèces ;

- $\quad$ le fruit possède 2 à 3 valves chez $C$. tridens L., 3 chez C. aestuans L. et $C$. fascicularis L., 3 à 4 chez $C$. trilocularis L., 5 chez C. olitorius var olitorius L. et 5 à 6 chez $C$. olitorius var incisifolius L. ; la section du fruit (PL.VII) est hexagonale chez C. aestuans $L$, circulaire chez $C$. fascicularis Lam., pentagonale chez les deux variétés de $C$. olitorius $L$. et trigone chez $C$. tridens $\mathrm{L}$. et $C$. trilocularis $\mathrm{L}$. ; il est terminé par trois dents chez $C$. aestuans $\mathrm{L}$. et $C$. tridens $\mathrm{L}$. et par une pointe entière chez les autres espèces ; la présence de 6 ailes est caractéristique du fruit de $C$. aestuans L. ; - les graines sont contiguës dans les fruits de $C$. aestuans L. et de $C$. fascicularis Lam. et séparées dans les fruits des autres espèces.

Les principales différences micromorphologiques au stade adulte sont les suivantes :-

- le pétiole chez les deux variétés de C. olitorius L. n'est pubescent que sur le sillon situé à la face supérieure alors que chez les autres espèces il est entièrement pubescent ; 

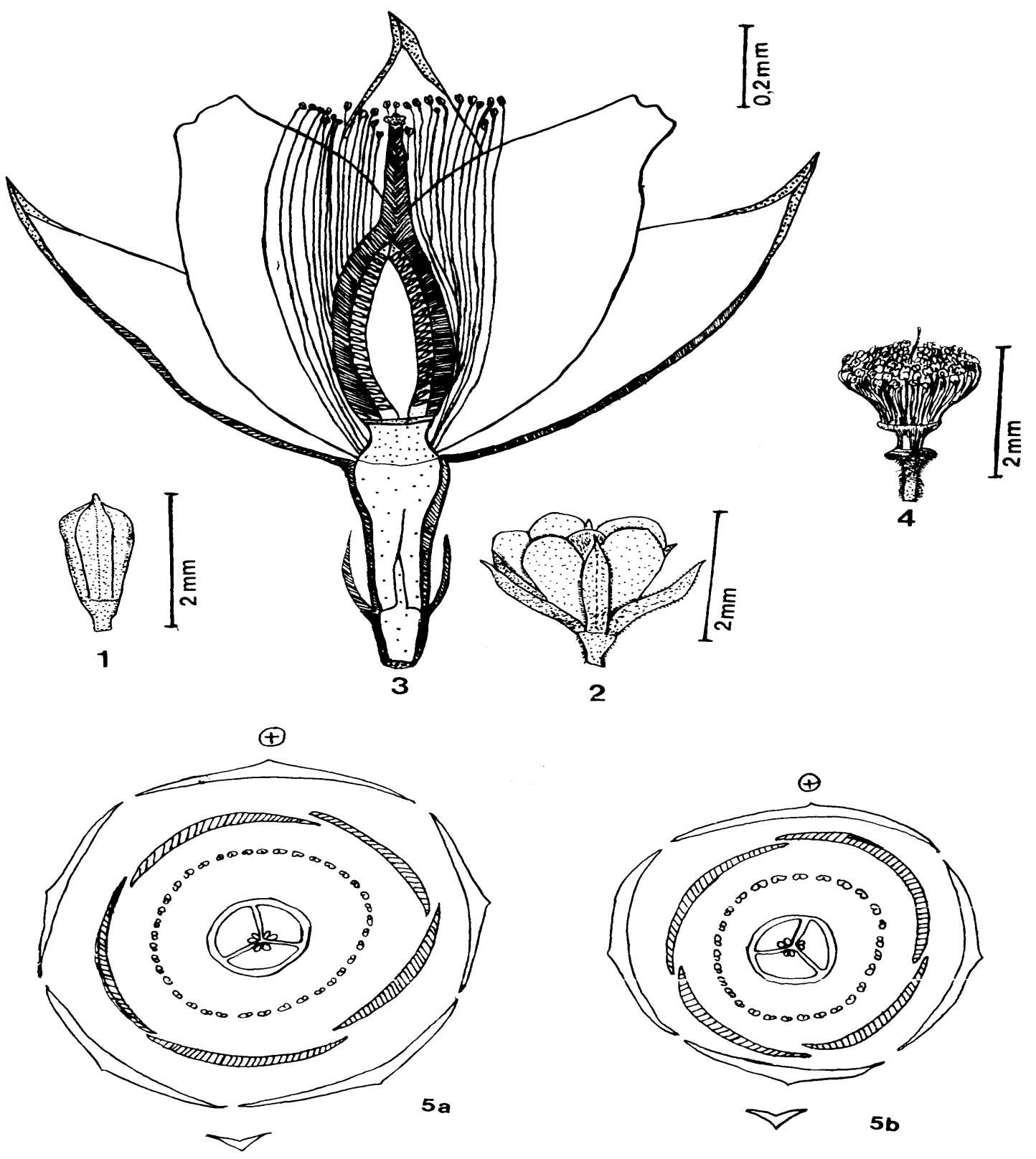

Planche V : Fleurs des différentes espèces

Fig.1 - Bouton floral ; Fig.2 - Fleur entière ; Fig.3 - Coupe Longitudinale de la fleur ; Fig.4 - Fleur sans le périanthe ; Fig.5 - Diagramme et Formule floraux ; $\mathrm{a}-C$. aestuans (fleur tétramère) ; b - Autres espèces (fleur pentamère)

- $\quad$ sur tous les organes pubescents, les poils tuberculés simples à soubassement vésiculeux ont été observés mais au niveau de la feuille; selon la localisation de ces poils on distingue d'une part des poils nervaires aux faces supérieure et inférieure des feuilles de $C$. aestuans L., de $C$. fascicularis L., de $C$. tridens L. et de $C$. trilocularis L. et seulement à la face inférieure des feuilles chez les variétés de $C$. olitorius L. et d'autre part des poils internervaires aux faces inférieure et supérieure des feuilles de $C$. aestuans L., de $C$. fascicularis Lam. et de $C$. trilocularis L. et uniquement à la face inférieure des feuilles de $C$. tridens L. ;

toutes les espèces présentent le même type stomatique (type anisocytique ou crucifère); cependant, les stomates de la face inférieure sont plus petits que ceux de la face supérieure chez les feuilles de $C$. aestuans L., des deux variétés de $C$. olitorius L. et de C. trilocularis L. alors qu'ils sont sensiblement de la 
Tableau IV : Caractères morphologiques du fruit et de la graine des espèces de Corchorus L.

\begin{tabular}{|c|c|c|c|c|c|c|c|}
\hline \multirow{2}{*}{\multicolumn{2}{|c|}{$\begin{array}{l}\text { Caractères du } \\
\text { fruit et de la } \\
\text { graine }\end{array}$}} & \multicolumn{6}{|c|}{ ESPECES } \\
\hline & & C. aestuans & C. fascicularis & \begin{tabular}{|l|} 
C. olitorius \\
var olitorius
\end{tabular} & $\begin{array}{l}\text { C. olitorius var } \\
\text { incisifolius }\end{array}$ & C. tridens & C. trilocularis \\
\hline \multirow{5}{*}{$\begin{array}{l}\mathrm{F} \\
\mathrm{R}\end{array}$} & Type & $\begin{array}{l}\text { Capsule à } \\
\text { déhiscence } \\
\text { loculicide }\end{array}$ & $\begin{array}{l}\text { Capsule à } \\
\text { déhiscence } \\
\text { loculicide }\end{array}$ & $\begin{array}{l}\text { Capsule à } \\
\text { déhiscence } \\
\text { loculicide }\end{array}$ & $\begin{array}{l}\text { Capsule à } \\
\text { déhiscence } \\
\text { loculicide }\end{array}$ & $\begin{array}{l}\text { Capsule à } \\
\text { déhiscence } \\
\text { loculicide }\end{array}$ & $\begin{array}{l}\text { Capsule à } \\
\text { déhiscence } \\
\text { loculicide }\end{array}$ \\
\hline & Forme & cylindrique & cylindrique & cylindrique & cylindrique & cylindrique & Cylindrique \\
\hline & Valves & 3 & 3 & 5 & 5 à 6 & 2 à 3 & 3 à 4 \\
\hline & Pilosité & Glabre & Subpubescent & Glabre & Glabre & Glabre & Pubescent \\
\hline & \begin{tabular}{|l|} 
Surface \\
interne
\end{tabular} & \begin{tabular}{|l|}
$\begin{array}{l}\text { Non ou à peine } \\
\text { cloisonné }\end{array}$ \\
\end{tabular} & $\begin{array}{l}\text { à peine } \\
\text { cloisonné }\end{array}$ & $\begin{array}{l}\text { Cloisonné et } \\
\text { favéolé }\end{array}$ & $\begin{array}{l}\text { Clois onné et } \\
\text { favéolé }\end{array}$ & à peine favéolé & $\begin{array}{l}\text { Profondément } \\
\text { favéolé }\end{array}$ \\
\hline \multirow{2}{*}{$\begin{array}{l}\text { U } \\
\mathrm{I}\end{array}$} & Section & Hexagonale & Circulaire & Pentagonale & pentagonale & trigone & Trigone \\
\hline & Sommet & 3 dents & $\begin{array}{l}\text { Bec court et } \\
\text { entier }\end{array}$ & $\begin{array}{l}\text { Bec long, } \\
\text { droit et entier }\end{array}$ & $\begin{array}{l}\text { Bec long, droit } \\
\text { et entier }\end{array}$ & 3 dents fines & $\begin{array}{l}\text { Bec court et } \\
\text { droit }\end{array}$ \\
\hline $\begin{array}{l}\mathrm{T} \\
\mathrm{S}\end{array}$ & Long. $(\mathrm{cm})$ & 1,5 à 2,5 & 1 à 2 & 2,5 à 8 & 2,5 à 8 & 3 à 5 & 4 à 8 \\
\hline $\mathrm{S}$ & $\begin{array}{l}\text { Nombre et } \\
\text { position }\end{array}$ & $\begin{array}{l}1 \text { à } 2 \text { opposés } \\
\text { au pétiole }\end{array}$ & $\begin{array}{l}\text { Fasciculés (3 à 8) } \\
\text { à la base du } \\
\text { pétiole }\end{array}$ & $\begin{array}{l}1 \text { à } 2 \text { opposées } \\
\text { au pétiole }\end{array}$ & $\begin{array}{l}1 \text { à } 2 \text { opposées } \\
\text { au pétiole }\end{array}$ & $\begin{array}{l}1 \text { à } 4 \text { opposés } \\
\text { au pétiole }\end{array}$ & $\begin{array}{l}2 \text { à } 3 \text { opposés } \\
\text { au pétiole }\end{array}$ \\
\hline & $\begin{array}{l}\text { Surface } \\
\text { externe }\end{array}$ & 6 ailes & $\begin{array}{l}\text { Lis se, glabre ou } \\
\text { pubescent }\end{array}$ & \begin{tabular}{|l} 
Stries \\
longitudinales \\
10 côtes
\end{tabular} & $\begin{array}{l}\text { Stries } \\
\text { longitudinales } \\
10 \text { côtes }\end{array}$ & Lis se ou strié & \begin{tabular}{|l} 
Stries \\
transversales 3 à \\
4 angles
\end{tabular} \\
\hline \multirow{2}{*}{$\begin{array}{l}\mathrm{G} \\
\mathrm{R} \\
\mathrm{A} \\
\mathrm{I}\end{array}$} & Forme & Polyédrique & Polyédrique & Pyramidale & Pyramidale & trapézoïdale & Polyédrique \\
\hline & Couleur & Marron à noire & Marron & $\begin{array}{l}\text { Marron } \\
\text { foncé à noire }\end{array}$ & $\begin{array}{l}\text { Marron foncé } \\
\text { à noire }\end{array}$ & Noire & Grise \\
\hline $\mathrm{N}$ & Disposition & Contiguës & contiguës & Séparées & Séparées & Séparées & Séparées \\
\hline \multirow{2}{*}{$\begin{array}{l}E \\
S\end{array}$} & Long. (mm) & 1 à 1,5 & 1 à 1,5 & 1 à 1,7 & 1,5 à 2 & 1 à 1,5 & 1 à 1,5 \\
\hline & Larg. (mm) & 0,5 à 1 & 0,5 à 1 & 1 à 2 & 2 à 2,5 & 0,5 à 1 & 0,5 à 1 \\
\hline
\end{tabular}

même taille sur les deux faces foliaires chez les autres espèces ; d'une manière générale, ils sont plus abondants à la face inférieure.

- les tiges sont glabres chez les deux variétés de $C$. olitorius $\mathrm{L}$. et pubescentes chez les autres espèces ;

A partir de ce tableau les suggestions suivantes sont faites pour améliorer la clé de détermination des espèces de Corchorus L. au Sénégal.

Plantes annuelles parfois pérennes avec des feuilles alternes, à marges dentées et poilues à nervation pennée arquée

Corchorus $L$.

. $\quad$ Feuilles sans filaments capillaires à la base du limbe.....C. fascicularis Lam.

. $\quad$ Feuilles avec deux filaments capillaires à la base du limbe

§ Feuilles triangulaires à marge lacérée...C. olitorius var incisifolius Asch. \& Schw.
$\S \quad$ Feuilles non triangulaires à marge régulièrement dentée

- Sommet du limbe apiculé et pétiole à face inférieure glabre..C. olitorius var olitorius L.

- Sommet du limbe non apiculé, pétiole pubescent sur toute sa surface

v Capsule terminée par un bec court et droit indivis..C. trilocularis $\mathbf{L}$.

v Capsule terminée par un bec divisé en trois dents divergentes ;

$\varnothing \quad$ Capsule à 6 ailes, courte et grosse, limbe ovale arrondi

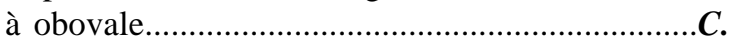
aestuans $\mathrm{L}$.

$\varnothing$ Capsule sans ailes, longue, mince, limbe ovale lancéolé à elliptique.

C. tridens L. 


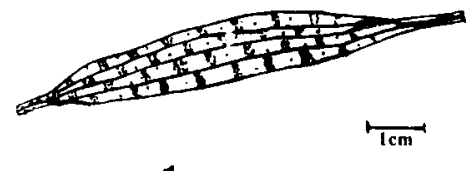

1

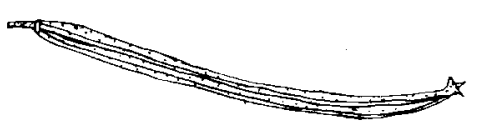

3

$$
\stackrel{\overrightarrow{0,6 \mathrm{~cm}}}{\overrightarrow{0}}
$$$$
-
$$

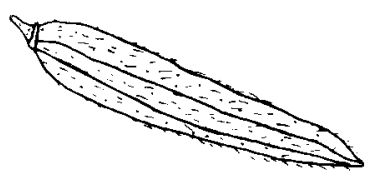

5

$\overrightarrow{0,3 \mathrm{~cm}}$

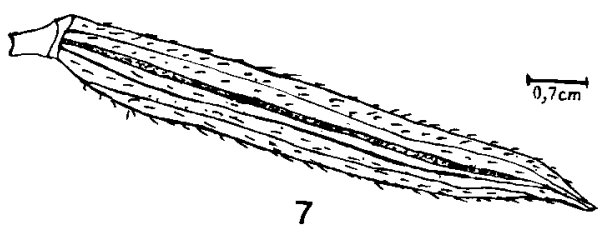

$\longdiv { 0 , 3 \mathrm { cm } }$

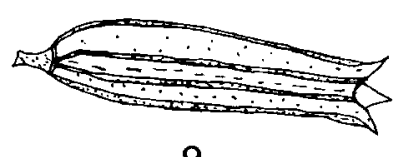

9
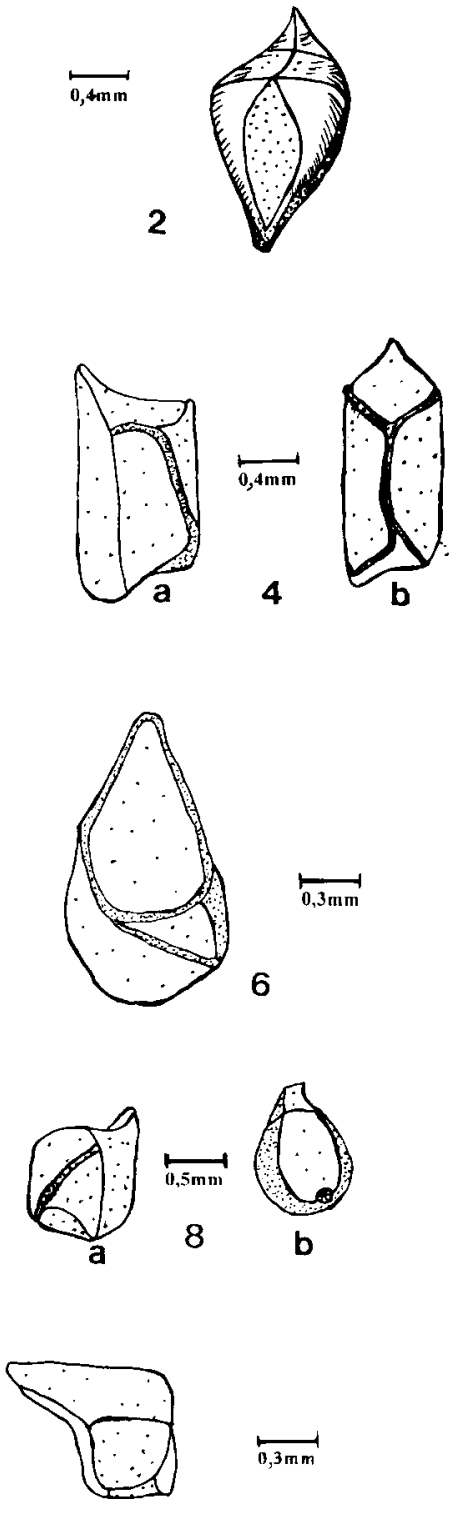

10

Planche VI : Fruits et graines des différentes espèces

Fig. 1 - Fruit des deux variétés de C. olitorius ; Fig. 2 - Graine des deux variétés de C. olitorius ; Fig. 3 - Fruit de C. tridens ; Fig. 4 - Graine de C. tridens (a et b) ; Fig.5 - Fruit de C. fascicularis ; Fig.6 - Graine de C. fascicularis ; Fig. 7 - Fruit de C. trilocularis ; Fig. 8 - Graine de C. trilocularis (a et b) ; Fig.9 - Fruit de C. aestuans ; Fig.10 - Graine de C. aestuans 
Tableau V : Tableau synoptique récapitulant les principaux caractères des espèces étudiées

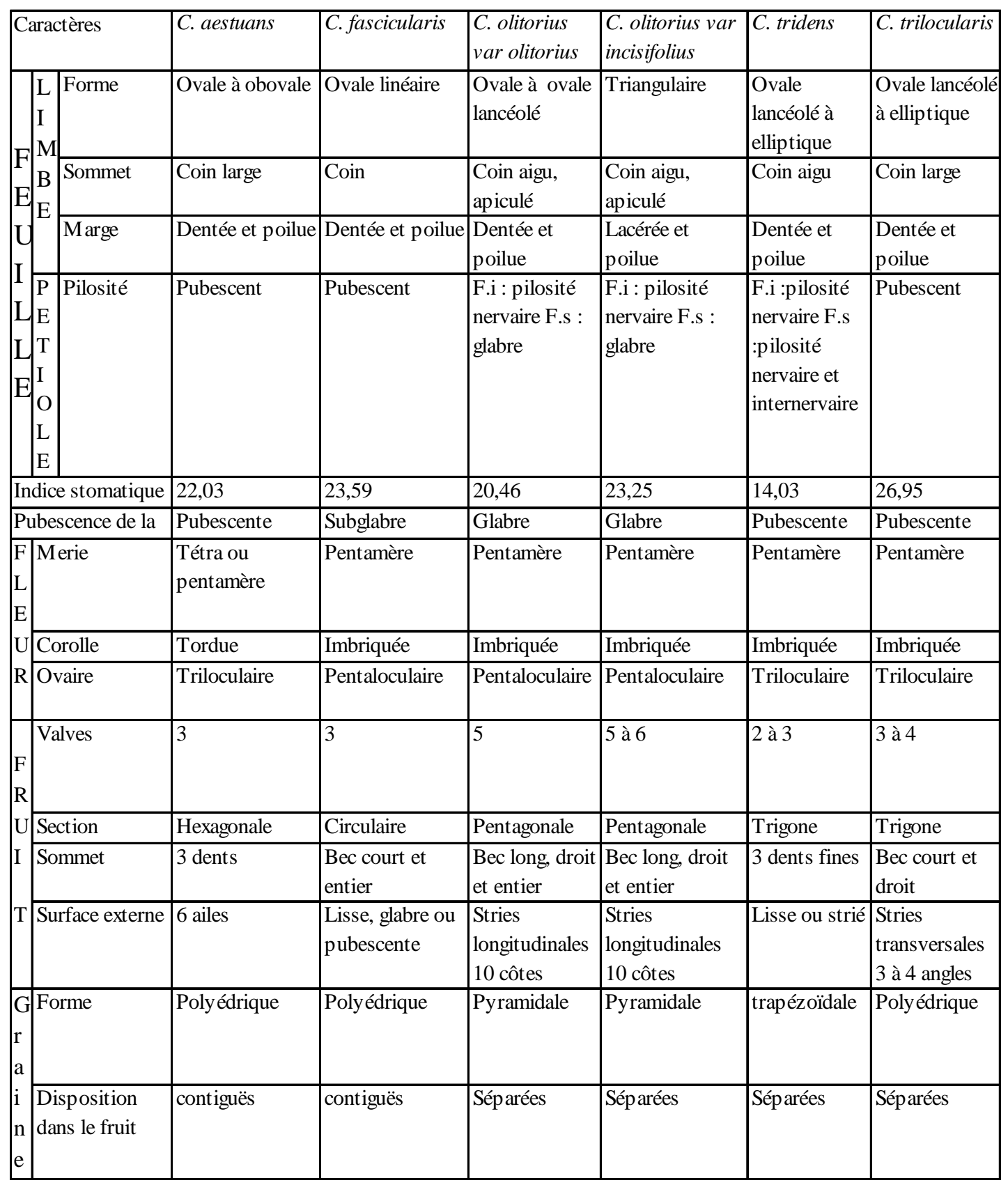



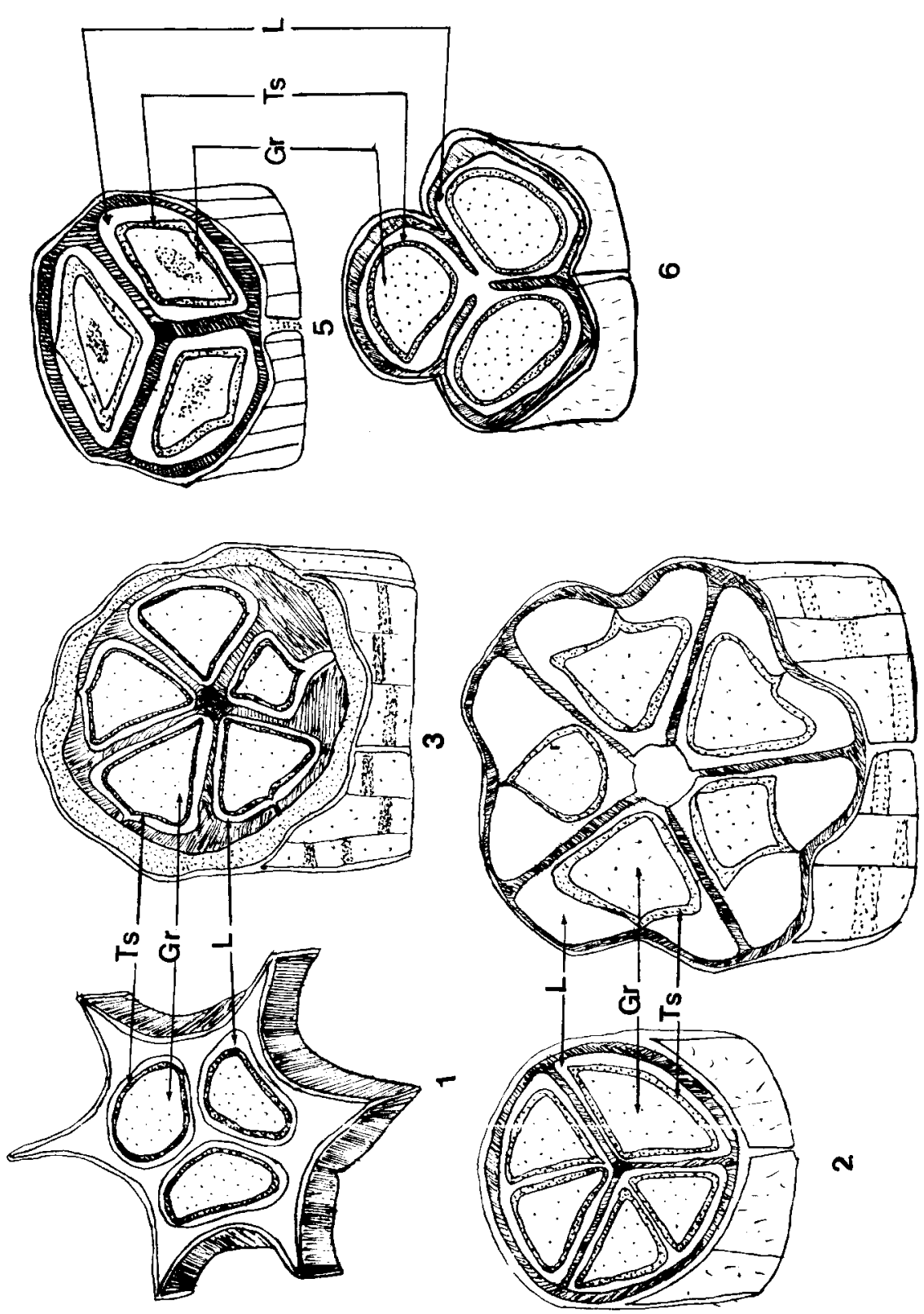

Planche VII : Coupes Transversales des fruits des espèces

Fig.1 - C. aestuans; Fig. 2 - C. fascicularis ; Fig.3 - C. olitorius var. olitorius ; Fig.4 - C. olitorius var. incisifolius ; Fig. 5 - C. tridens ; Fig. $6-$ C. trilocularis $;$ Gr. $=$ Graine $;$ L Loge carpellaire $;$ Ts $=$ Téguments séminaux 


\section{RÉFÉRENCESBIBLIOGRAPHIQUES}

AKORODA, M.O. (1988) - Cultivation of jute (Corchorus olitorius L.) for edible leaf in Nigeria. Trop. Agric. (Trinidad) 65 (4) : 297-299.

AKORODA, M.O. \& AKINTOBI, D.A. (1983) - Seed production in Corchorus olitorius L.. Acta Hortic. $123: 231-236$.

AKORODA, M.O. \& Olufajo, O.O. (1981) - Harvest index of market morphotypes of Corchorus olitorius L. at Ibadan, Nigeria. Abstracts, $6^{\text {th }}$ African Horticultural Symposium, Ibadan, Nigeria.

Anonyme, (1991) - Memento de l'agronome. ministère de la Coopération et du Dévelopement, République Française. pp1036-1038.

AYENSU, S.E. (1978) - medicinal plants of West Africa. Ref. Publ. Inc. p 259.

BAILLON, M.H. (1886) - Dictionnaire de Botanique (Tome II). Librairie Hachette et $\mathrm{C}^{\text {ie }}$; Paris. 207-208

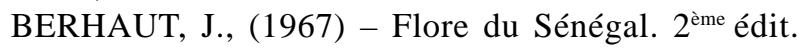
Clairafrique-Dakar, $485 \mathrm{p}$.

DUPONT, S. (1968) - révision des caractères des épidermes et des plantules chez les Mésembryanthémacées. Systématique. Evolution. Thèse d'Univ. Paul Sabatier, Toulouse.

EPENHUIJSEN, C.W. van (1974) - Growing native vegetables in Nigeria. F.A.O., Rome.

FAFUNSO, M. \& BASSIR, O. (1975) - Effects of age of some tropical vegetables on the amino acid composition of their extracted proteins W.A.J. biol. Applic. Chem. 18 : 17-20.

GORENFLOT, R. (1971) - Intérêt taxonomique et phylogénique des caractères stomatiques (application à la tribu des Saxifragacées). Boisseria, 19 : 181-195.

GORENFLOT, R. \& MOREAU, F. (1970) - Types stomatiques et phylogénie des Saxifraginées (Saxifragacées). C.R. Acad. Sc. Paris. 270 : 2802-2805.

GUYOT, M. (1966) - Les stomates des Ombellifères. . Bull. soc. Bot. Fr., 113 : 244-276.

HUTCHINSONP., DALZIEL J.M., KEAY R.W.J. \& HEPPER F.N., (1958)- Flora of West Tropical Africa. Vol I Part 2. $2^{\text {nd }}$ éd. Whitefriars Press Ltd, London, Tonbridge, England, 828p

JOHNSON, D.E. (1997) - Les adventices en riziculture en Afrique de l'Ouest (Weeds of rice in West Africa). Imprint Design, United Kingdom. pp 272-273

LEBOURGEOIS, TH. \& MERLIER, H. (1995)-Adventrop : les adventices d'Afrique soudano-sahélienne. CIRADCA Montpellier : pp 564-575 et pp 595- 615.

MBAYE M.S., (1999) - Contribution à l'étude biosystématique du genre Corchorus L. au Sénégal. Mémoire de DEA, Systématique végétale, Université Cheikh Anta Diop, faculté des sciences et techniques, Département de Biologie Végétale. Dakar, 86p.
MERLIER, H. \& MONTEGUT, J. (1982) - Adventices Tropicales. Ed. Ministère des Relations extérieures. Coopération et Développement. 490p.

METCALFE, C.R. \& CHALK, L. (1950) - Anatomy of the Dicotyledons. Clarendon press, Oxford (Volumes I et II). $1500 \mathrm{p}$.

MOREAU, F. (1979) - Etude morphologique et cytologique ders plantules de quelques Saxifragoïdées et Hydrangeoïdées ( Saxifragacées). Bull. soc. Bot. Fr., 126. $\mathrm{N}^{\circ} 3: 77-84$.

NOBA,. K. (1990) - Contribution à l'étude biosystématique de trois espèces du genre Boerhaavia (Nyctaginaceae) : B. diffusa L., B. erectaL., B. repens $L$. Thèse de Doctorat de $3^{\text {ème }}$ cycle de Biologie Végétale. Faculté des Sciences, Université de Dakar.

NOBA (K.) \& BA (A.T.), 1998. La végétation adventice du mil (Pennisetum typhoides Stapf et Hubbard) dans le Centre Ouest du Sénégal : étude floristique et phytosociologique. AAU Reports 39 : 113-125.

NOBA K, SAMB PI \& BA A.T. - Sur quelques caractères macro et micromorphologiques du jeune plant dans la systématique de trois espèces du genre Boerhavia L. (Nyctaginaceae). Bull Inst fond Afr noire Cheikh Anta Diop Dakar 1994 ; Ser A 47 : 51-62

NORMAN, J.C. (1992) - Tropical vegetable Crops. Arthur H. Stockwell Ltd. Elms Court Ilfracombe Devon. pp 203-208

OLIVIER-BEEVER, B. (1986)-Medicinal plants in Tropical West Africa. Cambridge University Press. pp 25, 262.

PURSGLOVE, J. W. (1968) - Tropical crops - Dicotyledons. Longman Group Limited. pp 613-619.

ROBERTY, G., (1954) - Petite flore de L'Ouest Africain. Ed. Larose.47- 48.

SALISBURY, E. J. (1927) - On the causes and ecological significance of stomatal frequency with special reference to the woodland flora. Phil. Trans. Royal. Soc. (London) B.241 1-65.

TIMMERMAN, H. A. (1927) - Stomatal number : Their value for distinguishing species Pharm. J. 118 : 241243 